\title{
Quantum mechanics investigation of perchloric acid decomposition releasing oxygen
}

Tanusree Chatterjee and Stefan T. Thynell

Department of Mechanical Engineering

The Pennsylvania State University, University Park, PA 16802

Geometries of reagents, TS, and products:

(Color code of atoms:- Red:Oxygen; Green:Chlorine; Gray:Hydrogen)

1. $\mathrm{HClO}_{4}$

$\mathrm{O}$

$\mathrm{Cl}$

$\mathrm{O}$

$\mathrm{O}$

$\mathrm{O}$

$\mathrm{H}$

2. $\mathrm{H}_{2} \mathrm{O}$

$\begin{array}{lrrr}\mathrm{O} & -0.00002000 & 0.11798100 & 0.00000000 \\ \mathrm{H} & 0.75904900 & -0.47081300 & 0.00000000 \\ \mathrm{H} & -0.75902900 & -0.47089100 & 0.00000000\end{array}$

3. $\mathrm{ClO}_{4}^{-}$

$\mathrm{O}$

$\mathrm{Cl}$

$\mathrm{O}$

$\mathrm{O}$

$\mathrm{O}$

$$
\begin{array}{rrr}
10.23074800 & -8.02253300 & 1.00101200 \\
11.10219000 & -7.14896800 & -0.06906200 \\
12.31242800 & -6.80598400 & 0.60047000 \\
10.22532100 & -6.05706000 & -0.29668500 \\
11.29698300 & -7.99466100 & -1.19928600 \\
10.76617500 & -8.80389100 & 1.23086000
\end{array}
$$

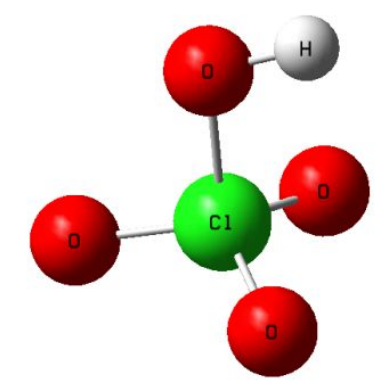




\section{4. $\mathrm{H}_{3} \mathrm{O}^{+}$}

$\begin{array}{lrrr}\mathrm{O} & 0.27322700 & 0.74096000 & 3.49271400 \\ \mathrm{H} & 0.48352100 & 1.66394200 & 3.26082500 \\ \mathrm{H} & 0.94383500 & 0.13719400 & 3.12429700 \\ \mathrm{H} & -0.61402500 & 0.50278800 & 3.16675300\end{array}$

5. $\mathrm{ClO}_{3}^{+}$
$\mathrm{O}$
$\mathrm{Cl}$
$11.94376600 \quad-7.25763500 \quad-1.35112800$
$\mathrm{O}$
$11.33870000 \quad-7.52417000 \quad-0.09963300$
$\mathrm{O}$
$11.52968700-8.77974200$
0.52553400
$\begin{array}{llll}10.54259300 & -6.53519800 & 0.52636800\end{array}$

\section{6. $\mathrm{O}_{3} \mathrm{ClOClO}_{3}$}

$\mathrm{O}$

$\mathrm{Cl}$

$\mathrm{O}$

$\mathrm{O}$

$\mathrm{O}$

$\mathrm{Cl}$

$\mathrm{O}$

$\mathrm{O}$

$\mathrm{O}$ $\begin{array}{llll}8.67774500 & -34.38349900 & -0.03750900\end{array}$

$\begin{array}{llll}6.66592600 & -34.25021800 & 2.63992000\end{array}$

$\begin{array}{llll}5.40463700 & -34.68620800 & 2.13696600\end{array}$

$\begin{array}{llll}8.94579100 & -36.74516200 & 0.38108500\end{array}$

$\begin{array}{llll}7.14563900 & -33.02333500 & 2.09204600\end{array}$

$\begin{array}{llll}8.07530300 & -35.61890700 & 0.34335800\end{array}$

$6.77694600-35.85306200-0.19987900$

$\begin{array}{llll}6.86944900 & -34.42863700 & 4.03791300\end{array}$

$\begin{array}{llll}7.82110200 & -35.47919800 & 2.09181400\end{array}$
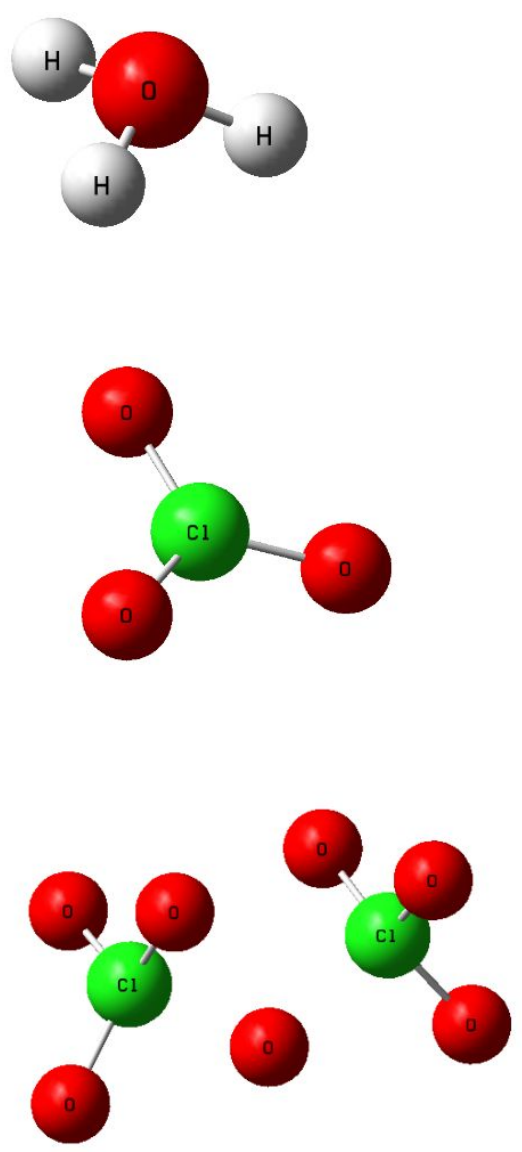

\section{7. $\mathrm{O}_{2} \mathrm{ClOOOClO}_{2}$}

$\mathrm{Cl}$

$\mathrm{O}$

$\mathrm{O}$

$\mathrm{O}$

$\mathrm{O}$

Cl

$\mathrm{O}$

$\mathrm{O}$

$\mathrm{O}$
$-2.13672900$

$-0.57682700$

$-1.18611300$

$-3.41055000$

1.25512500

1.72888200

1.79467000

0.0399220

2.9013240
0.29715500

$-1.48937200$

1.03017800

0.37539800

0.01739100

$-0.52790900$

$-0.15702100-0.24876200$

$-0.01772200 \quad 1.41980900$

$\begin{array}{ll}0.12461300 & 0.05311800\end{array}$

$1.49032800-0.43102600$

$-0.91426500-0.87961900$

$-0.67202100-0.25853800$

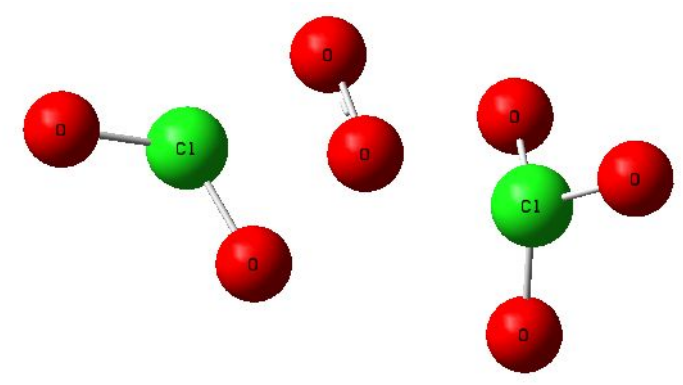


8. $\mathrm{O}_{2} \mathrm{ClOClO}_{2}$

$\begin{array}{lccc}\mathrm{Cl} & 6.14952100 & -34.19109900 & 2.17656900 \\ \mathrm{O} & 7.95523000 & -34.06532400 & 1.99915000 \\ \mathrm{O} & 5.98659700 & -33.04229600 & 3.04017500 \\ \mathrm{O} & 5.63468100 & -34.00910400 & 0.83779000 \\ \mathrm{Cl} & 8.81042500 & -35.16237900 & 0.82599100 \\ \mathrm{O} & 10.11362300 & -34.54569700 & 0.94134900 \\ \mathrm{O} & 8.15124600 & -34.93262700 & -0.44013700\end{array}$

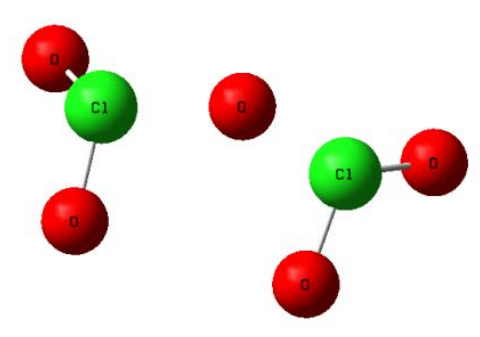

9. OCIOOOClO

$\begin{array}{lrrr}\text { Cl } & -2.04114100 & -0.82815700 & 0.17325700 \\ \mathrm{O} & 1.30857800 & -0.28578400 & -1.31332700 \\ \mathrm{O} & -1.30612500 & 0.16853800 & 1.23203600 \\ \mathrm{O} & -0.77853000 & 0.97509900 & -1.22668100 \\ \mathrm{Cl} & 1.71963100 & 0.09720200 & 0.12367600 \\ \mathrm{O} & 1.42144000 & -0.91463100 & 1.21948700 \\ \mathrm{O} & -0.48221800 & 1.44673900 & -0.14133300\end{array}$

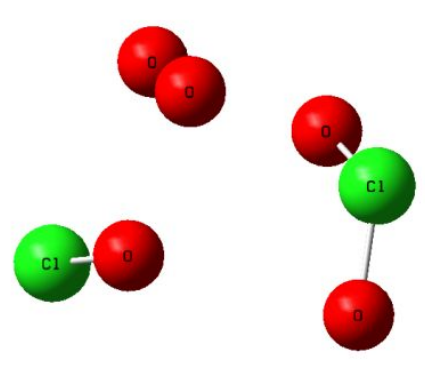

10. $\mathrm{ClOClO}_{2}$

$\begin{array}{llll}\mathrm{Cl} & 6.09593400 & -34.13356100 & 2.31728400 \\ \mathrm{O} & 8.03515900 & -34.37396900 & 2.28114400 \\ \mathrm{O} & 5.97448900 & -32.68951600 & 2.26126900 \\ \mathrm{O} & 5.70035500 & -34.83062400 & 1.10641200 \\ \mathrm{Cl} & 8.49069400 & -34.88355800 & 0.76976600\end{array}$

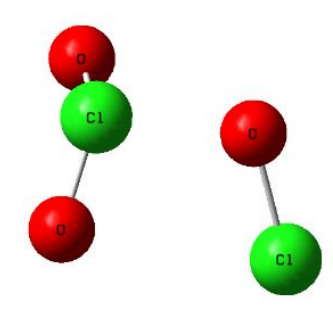

\section{ClOCl}

$\mathrm{Cl}$

$\begin{array}{llll}1.57924100 & -1.75899600 & -0.99791100\end{array}$

$\mathrm{O}$

$0.29629300-1.30136400$

0.03174000

$\mathrm{Cl}$

$\begin{array}{lll}-0.51453400 & -2.65827200 & 0.67755700\end{array}$

12. $\mathrm{ClOOCl}$

$\begin{array}{lrrr}\mathrm{Cl} & 0.57410700 & -0.50533000 & 1.54866000 \\ \mathrm{O} & -0.39381600 & -0.99654200 & 0.20429400 \\ \mathrm{Cl} & -0.44998600 & 1.15645700 & -1.20139100 \\ \mathrm{O} & 0.11597800 & -0.46123600 & -0.98165100\end{array}$

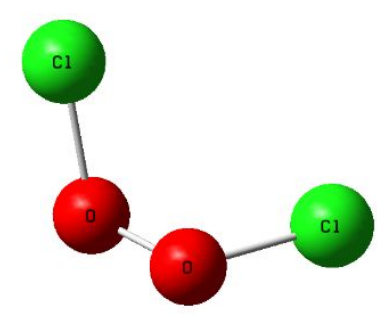


13. $\mathrm{O}_{2}$
$\mathrm{O}$
0.00000000
0.00000000
0.60284600
$\mathrm{O}$
0.00000000
$0.00000000-0.60284600$

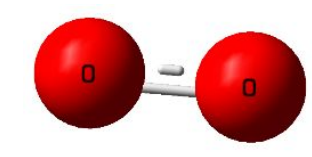

14. $\mathrm{Cl}_{2}$
$\mathrm{Cl}$
$\begin{array}{ll}-0.04881800 & 0.92354300\end{array}$
0.41498700
$\mathrm{Cl}$
$\begin{array}{ll}1.58946500 & -0.22862400\end{array}$
0.42266100

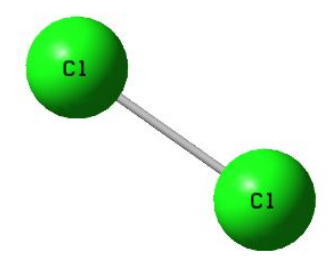

\section{TS_R2 $\left(2 \mathrm{HClO}_{4} \leftrightarrow \mathrm{O}_{3} \mathrm{ClOClO}_{3}+\mathrm{H}_{2} \mathrm{O}\right)$}

$\begin{array}{llrr}\mathrm{O} & 7.64274493 & -36.53736661 & -0.11642089 \\ \mathrm{Cl} & 6.37101761 & -33.40266287 & 2.28640576 \\ \mathrm{O} & 7.23302459 & -33.55164050 & 3.40110230 \\ \mathrm{O} & 6.74689646 & -32.57344962 & 1.19548630 \\ \mathrm{H} & 6.64736433 & -36.42688926 & 1.51781588 \\ \mathrm{O} & 6.19621803 & -36.07566929 & 2.30221298 \\ \mathrm{Cl} & 8.36642870 & -35.26943532 & -0.13753333 \\ \mathrm{H} & 5.28963842 & -36.39451614 & 2.23811444 \\ \mathrm{O} & 7.71096980 & -34.33601278 & -1.02697143 \\ \mathrm{O} & 4.98608056 & -33.68068841 & 2.43726624 \\ \mathrm{O} & 9.73975850 & -35.48228816 & -0.52671407 \\ \mathrm{O} & 8.35091608 & -34.71718404 & 1.23704781\end{array}$

16. TS_R3 $\left(2 \mathrm{HClO}_{4} \leftrightarrow \mathrm{O}_{2} \mathrm{ClOOOClO}+\mathrm{H}_{2} \mathrm{O}\right)$

$\begin{array}{lccc}\mathrm{O} & 10.53216800 & -8.36849900 & 1.44959900 \\ \mathrm{Cl} & 10.94804500 & -6.52336800 & -0.38395200 \\ \mathrm{O} & 12.14879700 & -6.37340400 & 0.39744700 \\ \mathrm{O} & 9.70465500 & -6.08020800 & 0.19173900 \\ \mathrm{O} & 10.90205800 & -7.64625000 & -1.38053200 \\ \mathrm{H} & 11.27373100 & -8.95730300 & 1.61155200 \\ \mathrm{O} & 8.63211100 & -9.75559100 & -0.24605400 \\ \mathrm{Cl} & 9.34972600 & -10.12387800 & -1.46915900 \\ \mathrm{O} & 10.81183200 & -9.62707400 & -1.31825100 \\ \mathrm{O} & 8.76275800 & -9.49114900 & -2.63715000 \\ \mathrm{O} & 9.46164200 & -11.56717000 & -1.61462300 \\ \mathrm{H} & 9.81140900 & -8.94681400 & 1.16834700\end{array}$
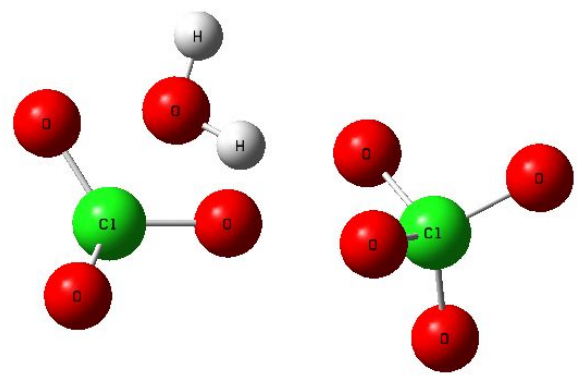


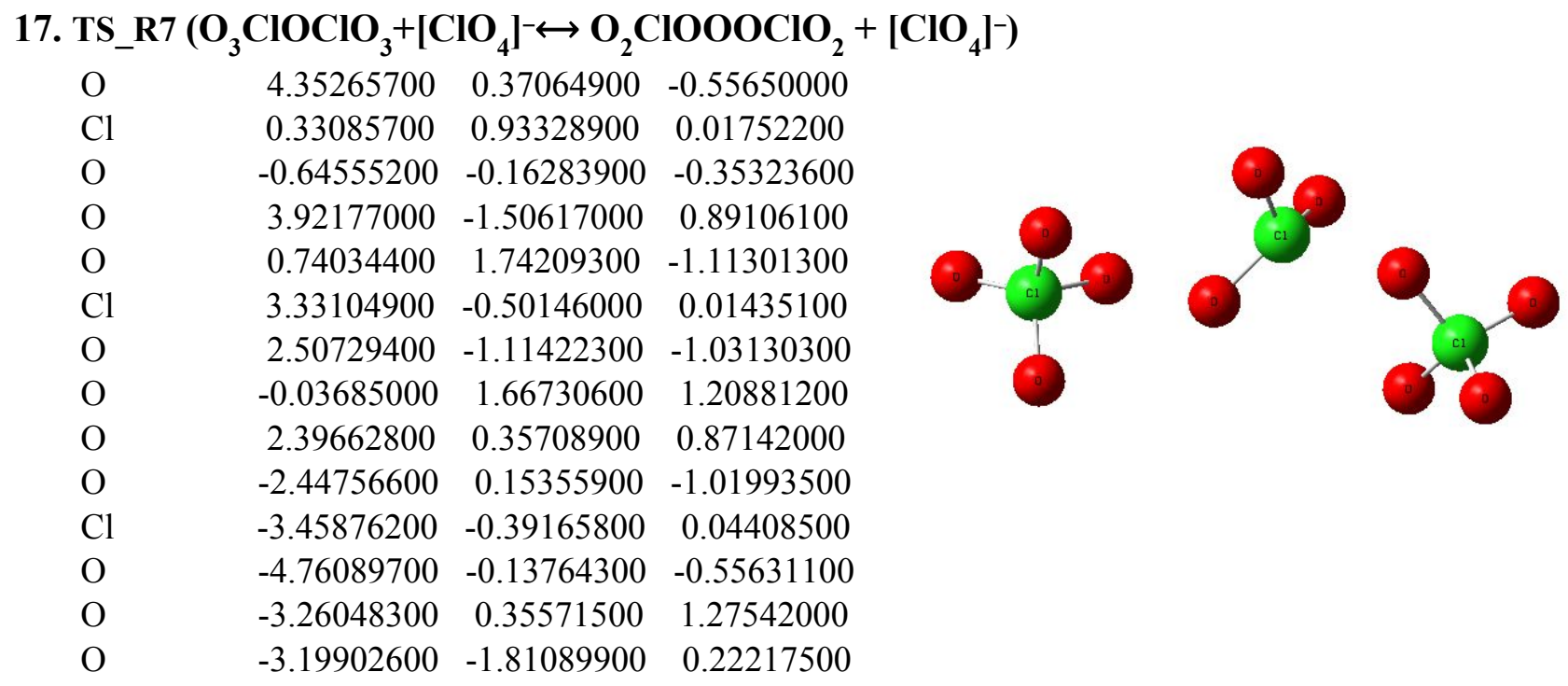

18. TS_R8 $\left.\left(\mathrm{O}_{3} \mathrm{ClOClO}_{3} \leftrightarrow \mathrm{O}_{2} \mathrm{ClOOOClO}\right)_{2}\right)$

$\begin{array}{llll}\mathrm{O} & 8.91537400 & -34.59890500 & -0.36595200 \\ \mathrm{Cl} & 6.83412900 & -34.29798700 & 2.71812400 \\ \mathrm{O} & 5.74407600 & -34.99077500 & 1.92076600 \\ \mathrm{O} & 8.68616100 & -36.93599200 & 0.42123800 \\ \mathrm{O} & 6.83221400 & -32.89149400 & 2.40879600 \\ \mathrm{Cl} & 8.07516900 & -35.74298500 & -0.11118400 \\ \mathrm{O} & 6.63420300 & -35.54550400 & 0.06709300 \\ \mathrm{O} & 6.55813200 & -34.54996200 & 4.11116300 \\ \mathrm{O} & 8.10307800 & -34.91461900 & 2.31566900\end{array}$

19. TS_R9 $\left(\mathrm{O}_{2} \mathrm{ClOOOClO}_{2} \leftrightarrow \mathrm{O}_{2} \mathrm{ClOClO}_{2}+\mathrm{O}_{2}\right)$

$\begin{array}{lrrr}\mathrm{O} & 1.57252700 & 1.15762800 & -1.40140100 \\ \mathrm{Cl} & -0.74110700 & 0.88588600 & 1.56609600 \\ \mathrm{O} & -0.88656400 & -1.92586100 & 0.06581400 \\ \mathrm{O} & 1.20714400 & -1.28430700 & -1.18492400 \\ \mathrm{O} & -0.84249600 & 0.99064500 & 0.01599400 \\ \mathrm{Cl} & 0.70163800 & 0.01506600 & -1.60946500 \\ \mathrm{O} & -1.41273500 & -1.14830300 & -0.69700800 \\ \mathrm{O} & 0.13981400 & 1.97224100 & 2.00920500 \\ \mathrm{O} & -0.08886900 & -0.42438200 & 1.82342800\end{array}$
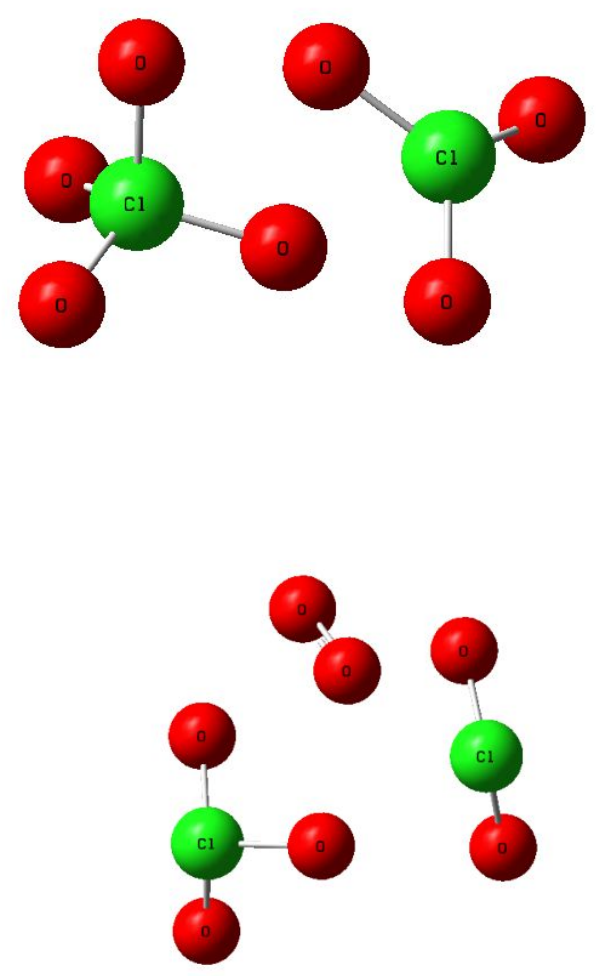


\section{TS_R10 ( $\mathrm{O}_{2} \mathrm{ClOClO}_{2} \leftrightarrow$ OClOOOCIO)}

$\mathrm{Cl}$

$\mathrm{O}$

$\mathrm{O}$

$\mathrm{O}$

$\mathrm{Cl}$

$\mathrm{O}$

$\mathrm{O}$ $\begin{array}{lll}-1.92117700 & -0.24475800 & -0.13878100\end{array}$

$\begin{array}{lll}1.07753300 & -1.30121700 & -0.60043200\end{array}$

$\begin{array}{lll}-2.85761000 & 0.05519400 & 0.97481600\end{array}$

$\begin{array}{lll}-0.89843800 & 0.89980300 & -0.46916400\end{array}$

$\begin{array}{lll}2.68494800 & -0.00502400 & 0.68943300\end{array}$

$\begin{array}{lll}0.46911400 & 0.87343600 & 0.53982700\end{array}$ $\begin{array}{lll}1.67273400 & -0.00083100 & -0.35437400\end{array}$
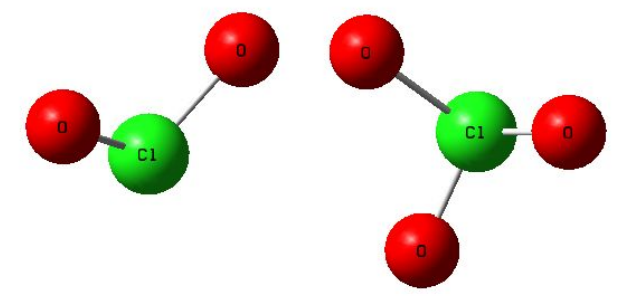

21. TS_R11 (OCIOOOCIO $\left.\leftrightarrow \mathrm{ClOClO}_{2}+\mathbf{O}_{2}\right)$

$\mathrm{Cl}$

$\begin{array}{lll}1.46462100 & -1.09254100 & 0.33280300\end{array}$

$\mathrm{O}$

$\begin{array}{rrr}-0.81718500 & 0.54927900 & 1.27094800\end{array}$

$\begin{array}{lll}0.55878100 & -0.76931600 & -0.95087400\end{array}$

$\begin{array}{llll}\text { O } & 1.41386300 & 1.43228600 & 0.24957400\end{array}$

$\begin{array}{lllll}\mathrm{Cl} & -1.34915000 & 0.34737300 & -0.07699900\end{array}$

$\begin{array}{llll}\mathrm{O} & -2.11238700 & -0.87806900 & -0.29723900\end{array}$

$\begin{array}{lllll}\mathrm{O} & 0.74750600 & 1.33223900 & -0.80811000\end{array}$

22. TS_R12 $\left(\mathrm{ClOClO}_{2} \leftrightarrow \mathrm{ClOCl}+\mathrm{O}_{2}\right)$

$\mathrm{Cl} \quad 6.22987400 \quad-34.00675100 \quad 1.80006800$

$\begin{array}{llll}\mathrm{O} & 6.03156400 & -34.88554300 & 2.97264000\end{array}$

$\begin{array}{llll}\mathrm{O} & 7.37820500 & -33.06145800 & 1.85245700\end{array}$

$\begin{array}{llll}\mathrm{Cl} & 10.36189800 & -34.72044700 & 1.10371700\end{array}$

$\begin{array}{llll}\mathrm{O} & 8.77133800 & -34.50130200 & 0.91413100\end{array}$

23. TS_R13 $\left(\mathrm{ClOCl}+\mathbf{C l O C l} \leftrightarrow \mathbf{C l O O C l}+\mathbf{C l}_{2}\right)$

\begin{tabular}{|c|c|c|c|}
\hline $\mathrm{Cl}$ & 0.26097300 & 1.66513100 & 0.07728100 \\
\hline $\mathrm{O}$ & -1.31215200 & 1.87198300 & 0.15532100 \\
\hline $\mathrm{Cl}$ & -2.32396100 & -0.41928900 & -0.18 \\
\hline $\mathrm{Cl}$ & -0.38040000 & -1.17407600 & 0.07 \\
\hline $\mathrm{O}$ & 1.55742700 & -1.36103000 & 0.52938200 \\
\hline $\mathrm{Cl}$ & 2.43799700 & -0.32593100 & -0.3719820 \\
\hline
\end{tabular}

24. TS_R14 (ClOOCl $\left.\leftrightarrow \mathrm{Cl}_{2}+\mathrm{O}_{2}\right)$

$\mathrm{Cl} \quad 0.65124500 \quad-0.19587900 \quad 1.70813900$

$\begin{array}{llll}\mathrm{O} & -0.52943400 & -1.30284400 & -0.31400000\end{array}$

$\begin{array}{lllll}\mathrm{Cl} & -0.13487700 & 1.29107300 & -0.60848600\end{array}$
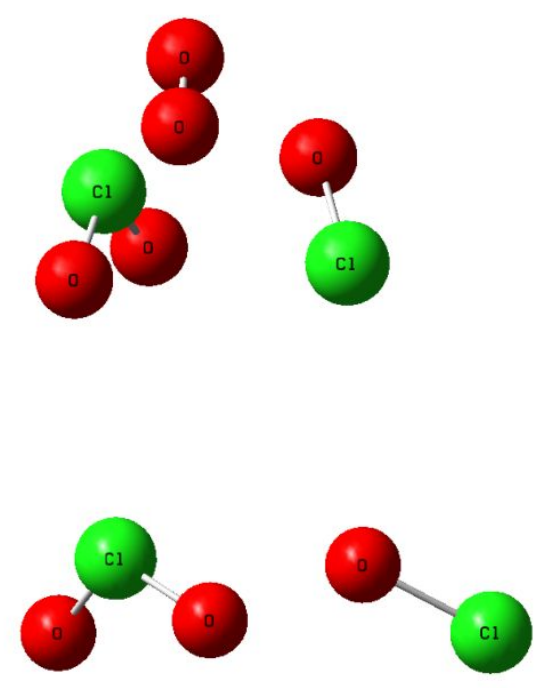

$\mathrm{O}$

$\begin{array}{lll}-0.14065000 & -0.59900200 & -1.21574200\end{array}$
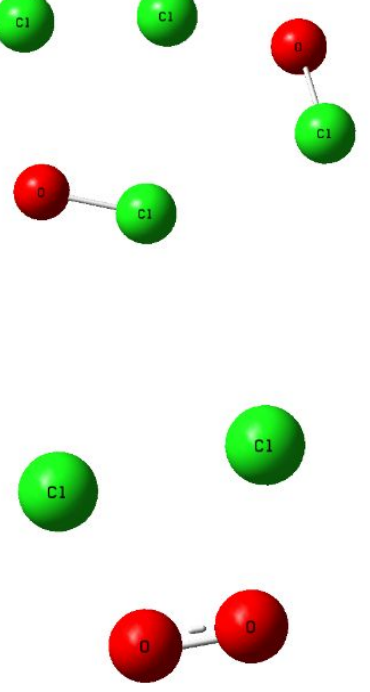
25. TS_R15 (ClOOCl $\left.+\mathrm{ClOOCl} \leftrightarrow 2 \mathrm{Cl}_{2}+\mathbf{2 \mathrm { O } _ { 2 }}\right)$

$\mathrm{Cl} \quad-0.01093700 \quad-0.04628500 \quad 2.88357500$

$\begin{array}{llll}\mathrm{O} & -0.14699600 & -1.07840900 & 1.07777800\end{array}$

$\begin{array}{llll}\mathrm{Cl} & -0.61545600 & 1.23799500 & -0.84635700\end{array}$

$\begin{array}{llll}\mathrm{O} & 0.47305600 & -0.49624400 & 0.17111100\end{array}$

$\begin{array}{llll}\mathrm{Cl} & 0.07578100 & 1.37863600 & 4.67983900\end{array}$

$\begin{array}{llll}\mathrm{O} & 0.79054800 & 3.15746400 & 3.43606300\end{array}$

$\begin{array}{llll}\mathrm{Cl} & -0.32706400 & 2.66744900 & 0.92501500\end{array}$

$\begin{array}{llll}\mathrm{O} & -0.06393300 & 3.70174500 & 2.71531200\end{array}$

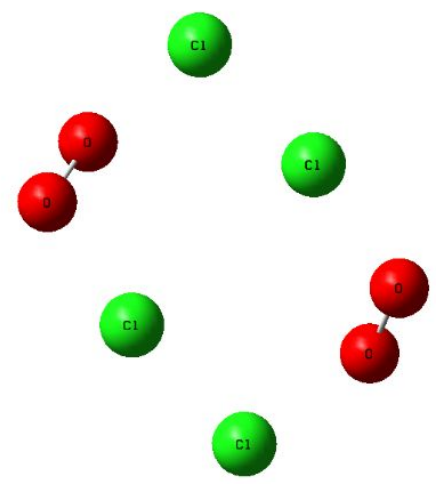




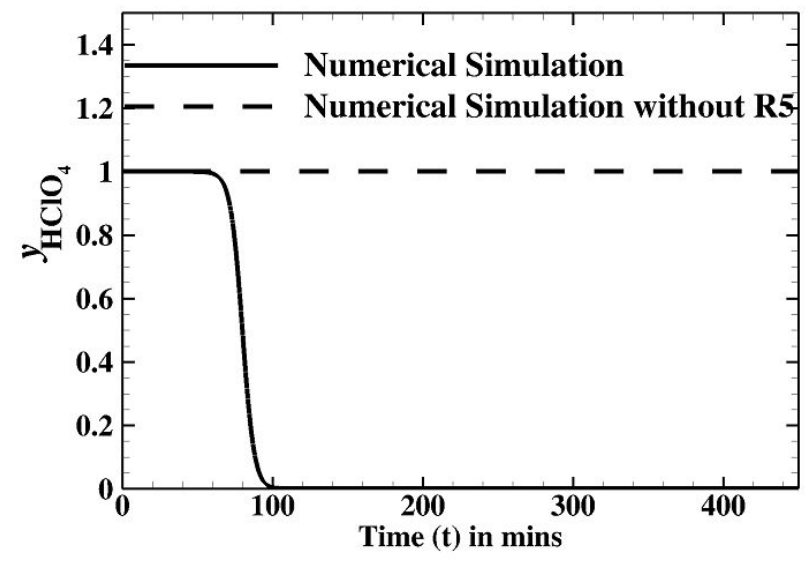

(a) $\mathrm{HClO}_{4}$

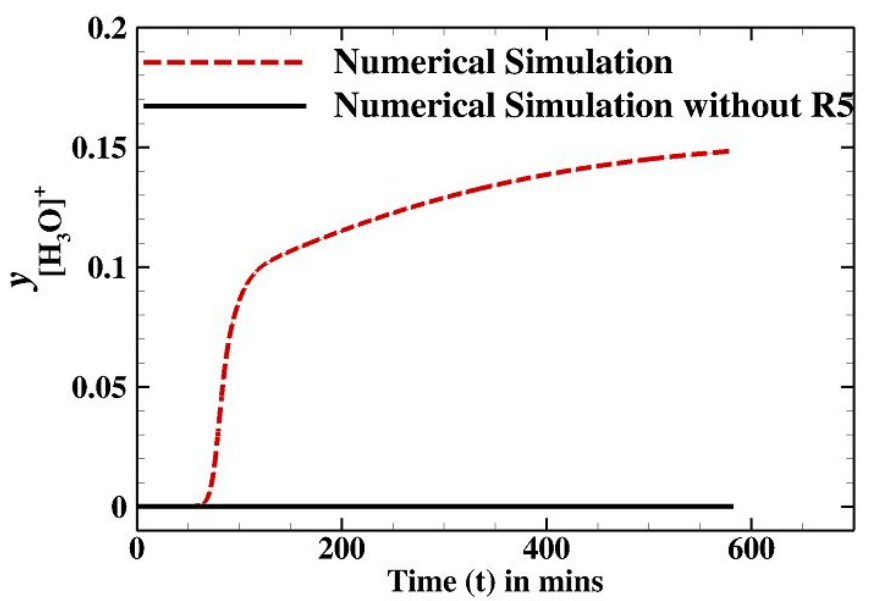

(c) $\left[\mathrm{H}_{3} \mathrm{O}\right]^{+}$

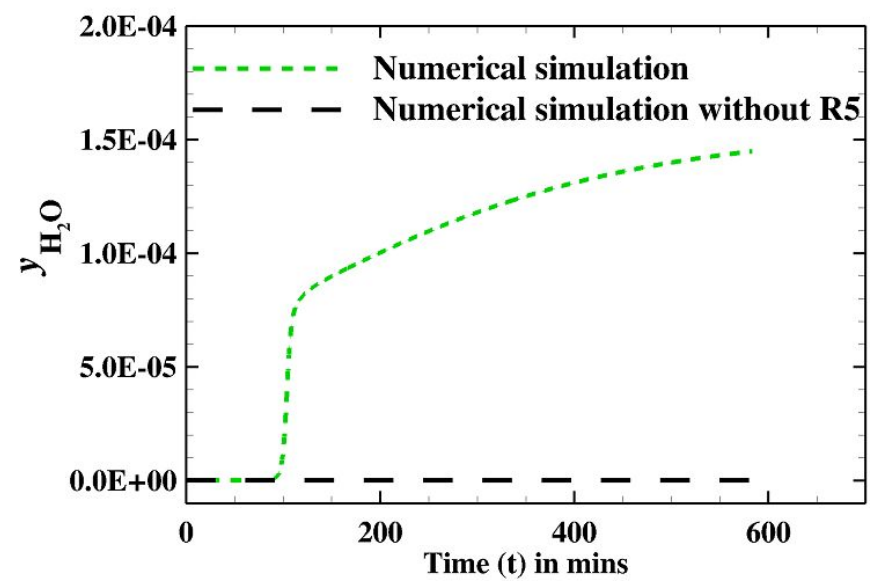

(b) $\mathrm{H}_{2} \mathrm{O}$

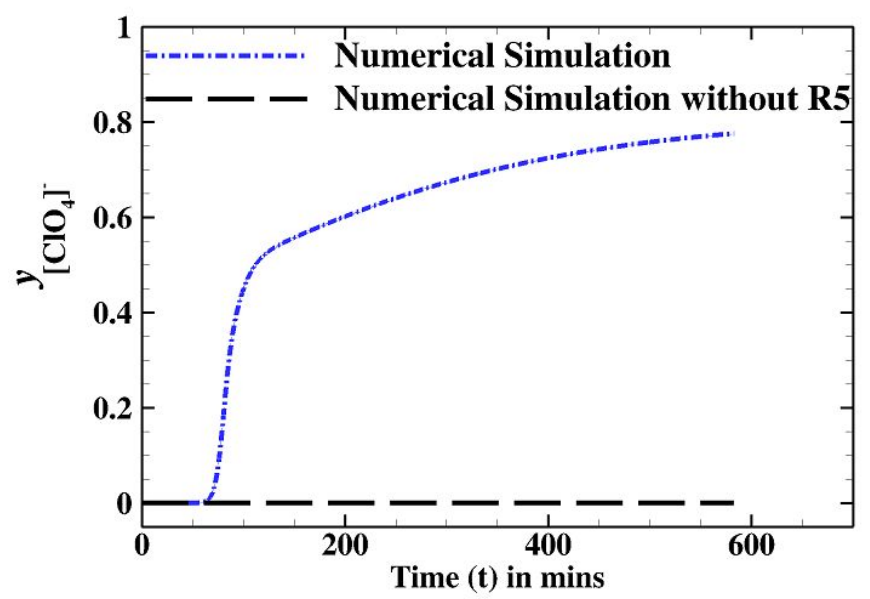

(d) $\left[\mathrm{ClO}_{4}\right]^{-}$

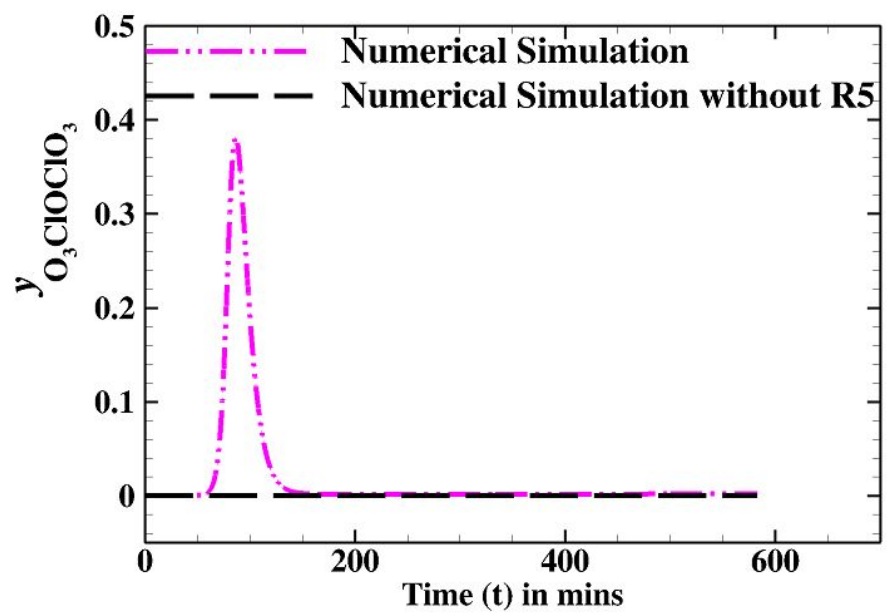

(e) $\mathrm{O}_{3} \mathrm{ClOClO}_{3}$

Fig. S1. Mass fraction $\left(y_{i}\right)$ of species $\left(\mathrm{HClO}_{4}, \mathrm{H}_{2} \mathrm{O},\left[\mathrm{ClO}_{4}\right]^{-},\left[\mathrm{H}_{3} \mathrm{O}\right]^{+}, \mathrm{O}_{3} \mathrm{ClOClO}_{3}\right)$ over time using the proposed mechanism with and without including reaction, $\mathbf{R 5}$ during simulation. Decomposition temperature, $T=353 \mathrm{~K}$ and initial mass fraction of anhydrous perchloric acid, $y_{\mathrm{HClO}_{4}, 0}=1$. 